\section{The Finnish EMBnet node: AGM 2010 report}

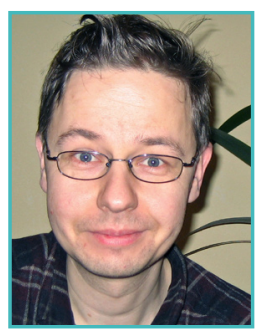

\section{Kimmo Mattila}

CSC - IT Center for Science, Espoo, Finland

CSC - IT Center for Science (http://www.csc.fi) is a non-profit company owned by the Finnish Ministry of Education, Science and Culture. With a staff of about 200 persons, CSC provides a wide range of information technology support and resources for the Finnish academic and research institutes. Currently, the services include computing, application, networking and data services for a wide variety of sciences, ranging from linguistics to physics.

\section{Bioinformatics services}

Bioscience researchers have for a long time been the largest user group of CSC. In 2009, 345 bioscientists used the computing servers of CSC, and approximately 200 more used applications developed or licensed by CSC. The bioinformatics software and databases, installed on the servers of CSC, form the core of our bioinformatics services. The application palette contains sequence databases, tools for sequence analysis, phylogenetics, gene mapping, DNA microarray analysis and structural biology. The support for next generation sequencing data analysis software is just emerging. The complete list of the over 50 available applications and databases can be found from the CSC- BioBox pages: http:// www.csc.fi/molbio. In addition to the instruction pages, CSC provides on-line support by e-mail and phone, and arranges bioinformatics related training courses and events.

At the moment, the main platform for the bioinformatics services is a HP ProLiant system with 64 computing cores. For heavier computing, CSC has two clusters and a Cray XT-4 supercomputer which together have over 15000 computing cores. Using the services of CSC requires, in most cases, a CSC user account. During the last few years, about 4-6 people have been actively maintaining and developing the bioinformatics services of CSC.

\section{Software development}

During the last years, CSC has invested significantly in software development, especially in the Chipster microarray data analysis tool (http:/l chipster.csc.fi/). Chipster brings a comprehensive collection of up-to-date analysis tools within the reach of bioscientists via its graphical user interface. The software was originally used for microarray data, but it is a generic tool and it now contains functionality also for proteomics, sequence analysis and next generation sequencing data.

\section{National and EU collaboration projects}

CSC is responsible for the National Grid Initiative (NGI) activities in Finland, and was one of the responsible organizations when the first significant grid research network, M-grid, for materials science, was being realized. CSC is also participating in several European Grid projects: these range from technology-oriented Grid projects, like EGEE2 and DEISA, to collaborative projects, like ELIXIR and EMBRACE. 